\title{
Ética das Relações Públicas: os casos da Public Relations Reviewe Journal of Public Relations Research
}

\author{
Public Relations Ethics: the cases of Public Relations Review \\ and Journal of Public Relations Research
}

Ética de las Relaciones Públicas: los casos de Public Relations Review y Journal of Public Relations Research

\section{Victor Silva Theodoro}

- Doutorando em Ciências da Comunicação pela Universidade da Beira Interior (UBI), Covilhã, Portugal

- Mestre em Comunicação Estratégica: Publicidade e Relações Públicas pela UBI

- Graduado em Relações Públicas pela Universidade Federal do Pampa (Unipampa)

- Investigador no Laboratório de Comunicação On-line (LabCom.IFP) - Centro de Investigação em Comunicação, Filosofia e Humanidades da UBI

- E-mail: victortheodoro1@hotmail.com

Gisela Marques Pereira Gonçalves

- Doutora em Ciências da Comunicação pela Universidade da Beira Interior, Covilhã, Portugal

- Professora Auxiliar do Departamento de Comunicação e Artes da UBI

- $\quad$ Presidente do Departamento de Comunicação e Artes da UBI

- Investigadora integrada no Laboratório de Comunicação On-line (LabCom.IFP) - Centro de Investigação em Comunicação, Filosofia e Humanidades da UBI

- E-mail: gisela.goncalves@labcom.ubi.pt 


\section{Resumo}

Este artigo tem como objetivo compreender a importância atribuída à investigação em ética nas revistas especializadas em relações públicas, em especial nas publicações Public Relations Reviewe Journal of Public Relations Research(2000-2015). Por meio do método bibliométrico, pode-se enumerar os artigos e categorias de acordo com o tema e perceber que, apesar da importância de estudar a ética das relações públicas, este ainda é um tópico pouco discutido nas principais revistas científicas especializadas nesse campo de investigação.

PALAVRAS-CHAVE: ESTUDO BIBLIOMÉTRICO •ÉTICA・RELAÇÕES PÚBLICAS.

\section{Abstract}

This article aims to understand the importance given to the research in ethics in journals specialized public relations, in particular, in the journals Public Relations Review and Journal of Public Relations Research (2000-2015). Through the bibliometric method, it is possible to enumerate the articles and categories according to the search topic and realize that, despite the importance of studying public relations ethics, this is still a search topic little discussed in the main scientific journals specialized in this field of research.

KEYWORDS: BIBLIOMETRIC STUDY • ETHICS•PUBLIC RELATIONS.

\section{Resumen}

Este artículo tiene como objetivo comprender la importancia atribuida a la investigación en ética en las revistas especializadas en relaciones públicas, en especial, en las publicaciones Public Relations Review y Journal of Public Relations Research (2000-2015). A través del método bibliométrico, se pueden enumerar los artículos y categorías relacionados con el tema y percibir que, a pesar de la importancia de estudiar la ética de las relaciones públicas, este, todavía, es poco discutido en las principales revistas científicas especializadas en ese campo de investigación. 
A prática profissional das relações públicas (RP) é objetivo de análise de artigos publicados em revistas científicas da área da comunicação. Desde o seu surgimento, as relações públicas fundamentam-se na ideia de mutualidade na comunicação, cuja principal função é o estreitamento de laços entre a organização e o público-alvo, buscando uma aproximação entre os interesses públicos e privados (Grunig, 1992).

0 contexto de formação de opinião - por meio da execução de ações de comunicação - e a aproximação desses interesses geram dilemas profissionais discutidos por teóricos da área (Gonçalves, 2007, 2013; Grunig; Hunt, 1984) que reiteram o papel das RP na construção de relacionamentos duradouros, transparentes e éticos.

A ética é vista como elemento fundamental para a institucionalização das RP como prática legítima nas organizações e na sociedade. Através dessa investigação, busca-se perceber se de fato há discussões referentes ao tema nas revistas $P$ ublic Relations Review (PRR) e Journal of Public Relations Research (JoPRR).

Os artigos relacionados com o tema da ética nas RP abarcam os mais variados conceitos e áreas de atuação na profissão, tais como: a ética na construção de ações de comunicação para os diversos públicos (Roper, 2005); a participação profissional à luz da ética na criação de ações de responsabilidade social (David; Kline; Dai, 2005); e por meio do desenvolvimento de um perfil ético padrão para a profissão (Lee, 2011).

Justifica-se a escolha dessas revistas como objeto de estudo pela elevada representatividade no cenário teórico/prático em que estão situadas e porque são as mais antigas da área das RP. De origem americana, são revistas internacionais que possuem uma periodicidade alta e constante, publicando trabalhos nas mais variadas vertentes da área. Também foram selecionadas devido a sua especialização no campo das RP (versus outras áreas das ciências da comunicação), a facilidade de acesso aos seus arquivos e, ainda, a sua proeminência nos debates acadêmicos e índices de citação.

A análise bibliométrica foi o método de pesquisa utilizado nesta investigação. Identificaram-se os principais temas trabalhados dentro da ética nas relações públicas, no contexto estudado e no período estabelecido: 2000-2015. Após a análise inicial, estipularam-se 19 categorias específicas a fim de perceber os principais conceitos-chave trabalhados dentro do universo estudado.

\section{ÉTICA DAS RELAÇÕES PÚBLICAS: BREVES CONSIDERAÇÕES}

Na sociedade contemporânea, as relações públicas possuem uma grande importância para o desenvolvimento institucional e mercadológico de uma organização. As ações e estratégias desenvolvidas pela área têm o intuito de gerar um ambiente harmônico e propício ao crescimento institucional. Segundo Grunig (1992), as relações públicas, por intermédio da gestão da comunicação, propiciam um contato mais amplo entre a organização e o seu público-alvo, efetivando assim sua principal função (comunicação simétrica).

A simetria comunicacional é entendida como o diálogo entre os interesses privados (organização) e o público (colaboradores, funcionários terceirizados e demais pessoas que possuem uma relação direta ou indireta com a empresa), na qual o feedback é real e ativo. Grunig (1992) afirma que as RP devem buscar uma compreensão mútua entre as partes, sob uma ideia de reciprocidade, isto é, no sentido comunitarista, a comunidade em que a organização está inserida deveria ser mais importante do que os interesses privados.

Por trabalhar diretamente com a aproximação de interesses privados e públicos, pelo seu papel na construção da opinião pública, uma comunicação regida por princípios éticos é necessária. 0 termo mutualidade na comunicação ganha destaque, 
priorizando bem-estar e equilíbrio no clima organizacional e, para isso, as opiniões e valores dos diversos públicos devem ser considerados para a construção e estabelecimento de metas organizacionais.

Fitzpatrick e Gauthier (2001), Gonçalves (2007; 2013), Gower (2003), Parsons (2004), entre outros autores, discutem sobre a importância da ética na prática profissional das RP, bem como a necessidade deste tema ser debatido com mais frequência.

Nesse sentido, discutir sobre a ética das RP se torna essencial para fundamentação da profissão. A ética busca nortear 0 papel a ser desempenhado por cada ser humano para o estabelecimento de uma sociedade mais equilibrada (Santos, 2012; Vázquez, 2007).

\section{MÉTODO E TÉCNICA DE PESQUISA}

Esta investigação visa apresentar resultados quantitativos por meio de uma análise bibliométrica das revistas Public Relations Reviewe Journal of Public Relations Research, no período de 2000 a 2015, a partir de publicações sobre o tema da ética nas relações públicas.

A revista Public Relations Review, fundada em 1975, disponibiliza um número maior de trabalhos e publica cinco volumes por ano, sendo quatro volumes gerais e um volume especial. Em contrapartida, o Journal of Public Relations Research, criado em 1989, possui um número médio anual de três a cinco volumes.

O estudo bibliométrico justifica-se como estratégia de análise por conseguir fundamentar - por meio de dados numéricos e porcentagens - os trabalhos publicados nas revistas no período estabelecido. E, por consequência, determinar as principais vertentes e categorias abordadas nesses artigos científicos.

Realizou-se uma pesquisa no site ScienceDirect ${ }^{1}$ com palavras-chave para restringir a busca, tais como: ethice public relations (vice-versa), deontological ethics e public relations (vice-versa), virtue ethics e public relations (vice-versa), entre outras.

O site disponibilizava também uma opção para acrescentar o nome da revista em análise e, neste caso, foram utilizadas as revistas Public Relations Review e Journal of Public Relations Research. Para a identificação das obras que incluíssem discussões prático-teórico-metodológicas sobre a ética nas RP, fez-se uma leitura dos títulos, resumos e palavras-chave de cada artigo publicado entre os anos de 2000 a 2015.

\section{CATEGORIZAÇÃO DOS TEMAS}

Para a efetivação das análises, optou-se pela construção de categorias que englobam os temas de estudos concentrados no universo da ética nas RP, de acordo com a pesquisa on-line em cada revista estudada.

Procedeu-se a uma análise dos trabalhos com o objetivo de identificar e segmentar os principais temas dos artigos sobre a ética nas RP e, a partir dessas informações, analisar os temas mais discutidos em ambas as revistas no período de 2000 a 2015. No total, foram criadas 19 categorias.

1 Disponível em: <http://www.sciencedirect.com/science/search> 
A categoria "Código de ética + Relações Públicas" contempla os trabalhos que se relacionam com a aplicação de um determinado código de ética, para entender a atuação das relações públicas ou pela necessidade da existência de um código para nortear o seu trabalho.

A segunda categoria, denominada "Ética + Modelos Relações Públicas", inclui trabalhos relacionados com os modelos de comunicação - agência de imprensa, informação pública, assimétrico bidirecional ou simétrico bidirecional - criados por Grunig e Hunt (1984).

A terceira, intitulada "Ética + Relações Públicas e Ensino Superior", envolve a análise de currículos (discente e docente) e da grade curricular para compreender a presença da ética no contexto universitário. Denominada "Ética + Relações Públicas e Audiovisual", a quarta categoria abarca as publicações referentes à ética das RP na construção de vídeos institucionais para a melhoria da imagem organizacional.

"Ética + Relações Públicas e Comunicação Estratégica" visa compreender todos os trabalhos que analisam a ética da profissão como uma função mais estratégica da comunicação. Estudos de caso relacionados com a participação das RP no conflito de crise e seus respectivos dilemas éticos também estão presentes nas publicações das revistas, estipulando assim a sexta categoria "Ética + Relações Públicas e Conflito de Crise".

As questões culturais que caracterizam os públicos organizacionais possibilitaram o acréscimo da categoria "Ética + Relações Públicas e Cultura", com artigos que buscam demonstrar a importância das RP na identificação do público-alvo, a fim de entender seu posicionamento no trabalho, seus objetivos e necessidades.

A oitava categoria, intitulada "Ética + Relações Públicas e Marketing", contempla as investigações que interpretam a utilização do marketing como ferramenta das RP à luz de questões éticas. "Ética + Relações Públicas e Profissionalismo", a nona categoria, envolve todas as atividades e funções desempenhadas pelas RP, desde: como a ética permeia 0 desenvolvimento do planejamento de comunicação, as ações de comunicação interna e externa, os dilemas éticos profissionais, entre outros pontos.

Na décima categoria, "Ética + Relações Públicas e Propaganda", opta-se por incluir as obras referentes à ética das RP no universo da propaganda, em um viés mais instrumental e persuasivo. Conceitos como responsabilidade social e sustentabilidade têm ganhado espaço nas discussões acadêmicas do século XXI. A décima primeira "Ética + Relações Públicas e Responsabilidade Social" vem para suprir tais abordagens e análises em um sentido ambiental e de responsabilidade com a sociedade.

Na décima segunda categoria, "Ética + Relações Públicas e Retórica", os autores buscam perceber como as RP fundamentam seu discurso no contexto organizacional. A décima terceira, "Ética + Relações Públicas e Turismo" transita sob uma área pouco explorada pelas RP.

Trabalhos que envolvam a relação existente entre RP e lobbyentram na décima quarta: "Ética + Relações Públicas e Lobby". Diante das funções exercidas por ambas as profissões, a ética se torna essencial para um crescimento/desenvolvimento mútuo entre a organização/governo e organização/públicos.

A décima quinta categoria, "Ética + Relações Públicas e Social Media", foi criada devido ao número de trabalhos publicados que relacionam o papel desempenhado pelo social media (ferramenta institucional). A décima sexta, "Ética + Relações Públicas e Comunicação Organizacional" engloba artigos relacionadas com o papel atribuido às relações públicas na gestão da comunicação. 
A décima sétima categoria, "Ética deontológica + Relações Públicas", aglomera trabalhos criados a partir da análise de uma ética deontológica. Na décima oitava, denominada "Ética + Relações Públicas e Feminismo", surgem artigos sobre a atividade das RP à luz da filosofia do movimento feminista. E, por fim, a décima nona, "Ética + Relações Públicas e Persuasão", é uma categoria que explicita o caráter persuasivo da profissão na construção das suas ações.

Essas categorias foram criadas para dar suporte às principais discussões e temas abordados no universo da ética das relações públicas no mundo acadêmico. Elas surgiram para suprir as principais atividades, ações e ferramentas desempenhadas pelas RP. A apresentação e discussão dos dados é a seguir exposta com base nessa categorização.

\section{ANÁLISE E DISCUSSÃO DOS RESULTADOS}

No total, identificaram-se 72 artigos relativos à ética das RP nas revistas estudadas.

Quadro 1: Número total de publicação nas revistas estudadas.

\begin{tabular}{|l|c|c|}
\hline \multicolumn{2}{|c|}{ Public Relations Reviewe Journal of Public Relations Research (2000-2015) } \\
\hline \multicolumn{1}{|c|}{ Nome do periódico } & Total de artigos publicados & Artigos sobre ética das RP \\
\hline Public Relations Review & 811 & 47 \\
\hline Journal of Public Relations Research & 298 & 25 \\
\hline Total & 1109 & 72 \\
\hline
\end{tabular}

Fonte: Elaborado pelos autores.

Por comparação com o universo total de artigos publicados entre 2000 e 2015, percebe-se que o número de trabalhos sobre ética nas RP é muito residual (Quadro 1): 47 artigos na Public Relations Review(PRR) (5,8\%) e 25 no Journal of Public Relations Research (JoPRR) (8,3\%). Ou seja, no universo da pesquisa (1109) apenas 72 (6,5\%) trabalham a temática.

Em seguida, analisou-se comparativamente os artigos publicados em cada revista em relação aos seguintes itens: artigos sobre ética por ano, artigos por país de origem do autor e artigos por categorias.

Em relação ao número de artigos publicados em cada revista, o primeiro ponto-chave a ser ressaltado é o aumento considerável do número de publicações sobre ética nos últimos cinco anos, principalmente na PRR. Entre os anos 2000 e 2009 houve um total de 18 (38,3\%) publicações nessa área na revista PRR, enquanto entre os anos 2010 e 2015 chegou ao número de 29 (61,7\%). Percebe-se que o acréscimo de 23,3\% decorreu, principalmente, pelo fato de que em 2011, $11(23,4 \%)$ trabalhos acoplavam a área estudada.

Em discrepância com os números obtidos na revista PRR, no JoPRR, o tema ética nas RP não possui uma grande representatividade em nenhuma edição/volume avaliado. Destacam-se os anos 2000, 2005 e 2015 com três artigos publicados em cada ano.

No JoPRR há um total de 28 universidades² envolvidas nas 25 publicações sobre o tema abordado. 0 número de publicações realizadas por pesquisadores americanos sobressai, com um total de 21 (84\%). Austrália (4\% - um), China (4\% - um) e Singapura (8\% - dois) totalizam quatro trabalhos neste período. 
Em relação às universidades de origem dos autores publicados na PRR, há um total de 47 universidades entre os anos 2000 e 2015, a maioria também de origem norte-americana (27-59,6\%). Os restantes artigos são autoria de investigadores sediados em instituições de Hong Kong, Palestina, África do Sul, Turquia, Suécia, Arábia Saudita, Vietnã, México, Coreia do Sul e Reino Unido. Não se identificaram artigos de autores sediados em universidades brasileiras em nenhuma das revistas.

Após as considerações iniciais da pesquisa, apresentam-se a seguir os dados relacionados com os temas/categorias mais abordadas em ambas as revistas.

Quadro 2: Número de publicações das categorias.

\begin{tabular}{|c|c|c|}
\hline \multicolumn{3}{|c|}{ Número de publicações das categorias (2000- 2015) } \\
\hline Temas & $\begin{array}{c}\text { Public Relations } \\
\text { Review }\end{array}$ & $\begin{array}{c}\text { Journal of Public } \\
\text { Relations Research }\end{array}$ \\
\hline Ética + Modelos Relações Públicas & 2 & 2 \\
\hline Ética + Relações Públicas e Comunicação Organizacional & 0 & 1 \\
\hline Ética + Relações Públicas e Feminismo & 0 & 1 \\
\hline Ética + Relações Públicas e Lobby & 0 & 1 \\
\hline Ética + Relações Públicas e Persuasão & 0 & 1 \\
\hline Ética + Relações Públicas e Profissionalismo & 21 & 12 \\
\hline Ética + Relações Públicas e Responsabilidade Social & 1 & 3 \\
\hline Ética + Relações Públicas e Retórica & 2 & 1 \\
\hline Ética + Relações Públicas e Social Media & 0 & 1 \\
\hline Ética deontológica + Relações Públicas & 1 & 2 \\
\hline Código de ética + Relações Públicas & 2 & 0 \\
\hline Ética + Relações Públicas e Ensino Superior & 8 & 0 \\
\hline Ética + Relações Públicas e Audiovisual & 1 & 0 \\
\hline Ética + Relações Públicas e Comunicação Estratégica & 1 & 0 \\
\hline Ética + Relações Públicas e Conflito de crise & 2 & 0 \\
\hline Ética + Relações Públicas e Cultura & 1 & 0 \\
\hline Ética + Relações Públicas e Marketing & 1 & 0 \\
\hline Ética + Relações Públicas e Propaganda & 3 & 0 \\
\hline Ética + Relações Públicas e Turismo & 1 & 0 \\
\hline Total & 47 & 25 \\
\hline
\end{tabular}

Fonte: Elaborado pelos autores.

O primeiro ponto a ser ressaltado é o número de publicações dentro da categoria "Ética + Relações Públicas e Profissionalismo". Em ambas as revistas é a categoria que possui maior representatividade: 21 (44,7\%) do total de publicações da PRR e 12 (48\%) no JoPRR, totalizando $33(45,8 \%)$ do universo. Por exemplo, na PRR há o artigo que trabalha com a ideia de consciência profissional a partir de Carl Jung (Fawkes, 2015) e no JoPRR o trabalho que avalia qualitativamente os dilemas éticos, que podem nortear o papel a ser desempenhado pelas relações públicas (Place, 2015). 
O número de publicações dentro da categoria "Ética + Relações Públicas e Ensino Superior", na Public Relations Review, também se destaca no Quadro 2 com um percentual de 17\% (8 publicações). Dentre os trabalhos, veja-se por exemplo o artigo de Hutchison (2002) que debate a necessidade do ensino da ética no ambiente universitário. Entretanto, não há nenhum trabalho publicado nesse período e nessa categoria no JoPRR.

Trabalhos que envolvem o universo da ética na propaganda (3 - 6,3\%), especificamente nas ações criadas por Ivy Lee, possibilitaram o surgimento de novos dilemas profissionais (St. John III, 2006). Esse trabalho exemplifica a categoria "Ética + Relações Públicas e Propaganda" no PRR. Tal como na categoria anterior, não há nenhum artigo sobre este tema no JoPRR.

Não há trabalhos no JoPRR que discutam os códigos de ética das relações públicas, e na PRR apenas dois artigos (4,2\%) abordam o tema. Como exemplo, veja-se como Watson (2014) contextualiza o surgimento do Código de Atenas e a sua importância para o estabelecimento de normas e padrões de conduta profissional.

A categoria "Ética + Relações Públicas e Conflito de crise", na PRR (2 -4,2\%), pode ser exemplificada com o artigo de Bowen (2015), em que apresenta o papel das relações públicas durante uma crise de imagem da Toyota. Não existem trabalhos publicados no JoPRR nesta nem nas quatro próximas categorias de análise: "Ética + Relações Públicas e Cultura", "Ética + Relações Públicas e Marketing", "Ética + Relações Públicas e Audiovisual" e "Ética + Relações Públicas e Comunicação Estratégica".

Dentro da categoria "Ética + Relações Públicas e Cultura" há um trabalho (2,1\%) publicado na PRR: El-Astal (2005) debate as mudanças culturais existentes nos diversos contextos sociais/organizacionais e como essas diferentes culturas influenciam a legitimidade das decisões das RP. Na categoria "Ética + Relações Públicas e Marketing" há apenas um trabalho (2,1\%) que apresenta as ações de marketing criadas pelas relações públicas de empresas localizadas na Turquia (Koc, 2006).

0 artigo de White (2012) é a única publicação (2,1\%) dentro da categoria "Ética + Relações Públicas e Audiovisual". Na categoria "Ética + Relações Públicas e Comunicação Estratégica" há apenas o artigo (2,1\%) de Lieber (2008) que analisa as diretrizes e ações de comunicação organzacional geridas pelas relações públicas.

Dentro do tema "Ética + Relações Públicas e Responsabilidade Social", na PRR, há apenas um artigo (2,1\%) que busca entender a participação das RP no desenvolvimento de ações de responsabilidade social (Munshi; Kurian, 2005). Esse tema esteve mais presente nas discussões do JoPRR, totalizando três artigos (12\%), como o trabalho dos autores Molleda e Ferguson (2004) que analisam o perfil profissional das relações públicas brasileiras tendo como base norteadora a ética e responsabilidade social ${ }^{3}$.

Dois artigos (4,2\%) foram identificados na PRR dentro da categoria "Ética + Relações Públicas e Retórica": Smudde e Courtright (2011) analisam a ética do discurso como uma função mediadora entre colaboradores e o alto escalão; e o artigo de Stokes e Waymer (2011), demonstra a necessidade da discussão da ética na retórica/discurso da profissão no ambiente universitário. Nessa mesma categoria, no JoPRR, o único trabalho publicado (4\%) foi de Heath (2000), que contextualiza a herança retórica das RP, bem como suas principais características com o passar dos anos.

Os autores L'Etang, Falkheimer e Lugo (2007) refletem criticamente sobre a ética profissional na área cultural, especificamente no turismo, possibilitando assim o surgimento da categoria "Ética + Relações Públicas e Turismo" na PRR (2,1\%). Mais uma vez, tal como em categorias anteriores já referidas, não há trabalhos publicados no JoPRR.

30 artigo centra-se na realidade brasileira, mas é produzido por autores sediados na Universidade da Flórida, nos Estados Unidos da América. 
Há dois trabalhos (4,2\%) publicados na PRR dentro da categoria "Ética + Modelos Relações Públicas". Como exemplo, citese 0 artigo de Brown (2006), que analisa os modelos de comunicação propostos por Grunig e Hunt (1984) à luz da ética. No JoPRR, há também dois trabalhos (8\%) publicados, como a investigação de Roper (2005), que critica a teoria simétrica da comunicação à luz do conceito de hegemonia.

Nas próximas quatro categorias não existem trabalhos publicados na PRR: "Ética + Relações Públicas e Feminismo", "Ética + Relações Públicas e Lobby", "Ética + Relações Públicas e Comunicação Organizacional" e "Ética + Relações Públicas e Social Media".

A categoria "Ética + Relações Públicas e Feminismo" emergiu na JoPRR com um único artigo (4\%), publicado por Grunig, Toth e Hon (2000), que realizam um estudo comparativo entre os principais fundamentos sobre a ética das relações públicas e os valores feministas. Também com um único trabalho (4\%), foi possível estabelecer a categoria "Ética + Relações Públicas e Lobby", em que Wise (2007) analisa as estratégias de lobby (da área da saúde) em um viés mais relacional, como as funções básicas das relações públicas.

A pesquisadora Auger (2014) analisa a ética e a transparência da comunicação organizacional como meios de qualificação e melhoria de reputação e imagem perante o público-alvo (4\%) ("Ética + Relações Públicas e Comunicação Organizacional"). 0 trabalho (4\%) de Sweetser (2010) é o único dentro da categoria "Ética + Relações Públicas e Social Media": discorre sobre os novos dilemas éticos que surgem através de ações de relacionamento organizacional nos social media.

Na última categoria estabelecida, denominada "Ética deontológica + Relações Públicas", há um trabalho (2,1\%) publicado na PRR, de autoria de Paquette, Sommerfeldt e Kent (2015), que analisam a prática das RP à luz de uma ética consequencialista. No JoPRR há dois trabalhos (8\%) de Bowen $(2004,2005)$. Em ambos a autora busca criar um modelo ético das RP com base na filosofia deontológica de Kant.

Apenas nas categorias "Ética + Modelos Relações Públicas", "Ética + Relações Públicas e Profissionalismo", "Ética + Relações Públicas e Responsabilidade Social", "Ética + Relações Públicas e Retórica" e "Ética deontológica + Relações Públicas" há trabalhos publicados em ambas as revistas no período estudado.

Em suma, pode-se constatar que pesquisas que envolvem a temática da ética nas relações públicas ainda são pouco frequentes nos últimos 15 anos em ambas as revistas. Esse fato pode ser comprovado tendo como base os dados analisados.

\section{CONSIDERAÇÕES FINAIS}

Para esta investigação, partiu-se do pressuposto de que estudar e analisar o universo da ética das relações públicas é fundamental para a institucionalização da prática profissional nos diversos contextos organizacionais.

Com base no estudo bibliométrico pode-se concluir que a discussão sobre a ética nas relações públicas ainda não possui uma grande representatividade no número total de artigos publicados na PRR e JPRR nos anos de 2000 a 2015.

As 19 categorias criadas representam as vertentes estudadas dentro do universo da análise. Contudo, acredita-se que pela importância das relações públicas na construção e formação da opinião pública, os dilemas e questões éticas deveriam ser foco de investigação, a fim de qualificar e legitimar a prática profissional nos seus diversos contextos de atuação. 
A ética não ocupa um lugar preponderante no universo de artigos das duas revistas de relações púbicas estudadas (apenas 6,5\% do total). Em estudos futuros, seria também importante analisar o tema da ética nas RP no seio das revistas especializadas em ética na comunicação, como o Journal of Business Ethics.

Este estudo demonstra que as publicações Public Relations Reviewe Journal of Public Relations Researchsão um importante suporte à difusão de trabalhos das RP e que poderão ser alvo de publicações por parte de investigadores brasileiros na área das RP em geral e da ética profissional em particular.

\section{REFERÊNCIAS}

AUGER, Giselle A. Trust me, trust me not: an experimental analysis of the effect of transparency on organizations. Journal of Public Relations Research, Abingdon, v. 26, n. 4, p. 325-343, 2014.

BOWEN, Shannon A. Expansion of ethics as the tenth generic principle of public relations excellence: a Kantian theory and model for managing ethical issues. Journal of Public Relations Research, Abingdon v. 16, n. 1, p. 65-92, 2004.

A practical model for ethical decision making in issues management and public relations. Journal of Public Relations Research, Abingdon, v. 17, n. 3, p. 191-216, 2005.

Auto recall crisis, framing, and ethical response: Toyota's missteps. Public Relations Review, Amsterdam, v. 41, n. 1, p. 40-49, 2015.

BROWN, Robert E. Myth of symmetry: public relations as cultural styles. Public Relations Review, Amsterdam, v. 32, n. 3, p. 206-212, set. 2006.

DAVID, Prabu; KLINE, Susan; DAI, Yang. Corporate social responsibility practices, corporate identity, and purchase intention: a dual-process model. Journal of Public Relations Research, Abingdon, v. 17, n. 3, p. 291-313, 2005.

EL-ASTAL, Mohammed A. S. Culture influence on educational public relations officers' ethical judgments: a cross-national study. Public Relations Review, Amsterdam, v. 31, n. 3, p. 362-375, set. 2005.

FAWKES, Johanna. A Jungian conscience: self-awareness for public relations practice. Public Relations Review, Amsterdam, v. 41, n. 5, p. 726-733, dez. 2015.

FITZPATRICK, Kathy; GAUTHIER, Candace. Toward a professional responsibility theory of public relations ethics. Journal of Mass Media Ethics, Abingdon, v. 16, n. 2-3, p. 193-212, 2001.

GONÇALVES, Gisela. Ética das relações públicas: a falta de responsabilidade social nos Códigos Éticos de Relações Públicas. Biblioteca On-line de Ciências da Comunicação, Covilhã, p. 1-29, 2007.

GONÇALVES, Gisela. Ética das relações públicas. Coimbra: Minerva Coimbra, 2013.

GOWER, Karla K. Legal and ethical restraints on public relations. Long Grove: Waveland Press, 2003. 
GRUNIG, James E. Excellence in public relations and communication management. New Jersey: Lawrence Erlbaum, 1992.

GRUNIG, James E.; HUNT, Todd. Managing public relations. New York: Holt, Rinehart \& Winston, 1984.

GRUNIG, Larissa A.; TOTH, Elizabeth L.; HON, Linda Childers. Feminist values in public relations. Journal of Public Relations Research, Abingdon, v. 12, n. 1, p. 49-68, 2000.

HEATH, Robert L. A rhetorical perspective on the values of public relations: crossroads and pathways toward concurrence. Journal of Public Relations Research, Abingdon, v. 12, n. 1, p. 69-91, 2000.

HUTCHISON, Liese L. Teaching ethics across the public relations curriculum. Public Relations Review, Amsterdam, v. 28, n. 3, p. 301-309, ago. 2002.

ST. JOHN III, Burton. The case for ethical propaganda within a democracy: Ivy Lee's successful 1913-1914 railroad rate campaign. Public Relations Review, Amsterdam, v. 32, n. 3, p. 221-228, set. 2006.

KOC, Erdogan. "Order three advertisements and get one news story free": public relations ethics practices of Turkish and international companies in Turkey. Public Relations Review, Amsterdam, v. 32, n. 4, p. 331-340, nov. 2006.

L'ETANG, Jacquie; FALKHEIMER, Jesper; LUGO, Jairo. Public relations and tourism: critical reflections and a research agenda. Public Relations Review, Amsterdam, v. 33, n. 1, p. 68-76, mar. 2007.

LEE, Seow Ting. Conceptualizing ethical knowledge and knowledge transfer in public relations. Public Relations Review, Amsterdam, v. 37, n. 1, p. 96-98, mar. 2011.

LIEBER, Paul S. Moral development in public relations: measuring duty to society in strategic communication. Public Relations Review, Amsterdam, v. 34, n. 3, p. 244-251, set. 2008.

MOLLEDA, Juan-Carlos.; FERGUSON, Mary Ann. Public relations roles in Brazil: hierarchy eclipses gender differences. Journal of Public Relations Research, Abingdon, v. 16, n. 4, p. 327-351, 2004.

MUNSHI, Debashish; KURIAN, Priya. Imperializing spin cycles: a postcolonial look at public relations, greenwashing, and the separation of publics. Public Relations Review, Amsterdam, v. 31, n. 4, p. 513-520, nov. 2005.

PAQUETTE, Michael; SOMMERFELDT, Erich J.; KENT, Michael L. Do the ends justify the means? Dialogue, development communication, and deontological ethics. Public Relations Review, Amsterdam, v. 41, n. 1, p. 30-39, mar. 2015.

PARSONS, Patricia J. Ethics in public relations: a guide to best practice. London: Kogan Page, 2004.

PLACE, Katie R. Exploring the role of ethics in public relations program evaluation. Journal of Public Relations Research, Abingdon, v. 27, n. 2, p. 118-135, 2015.

ROPER, Juliet. Symmetrical communication: excellent public relations or a strategy for hegemony? Journal of Public Relations Research, Abingdon, v. 17, n. 1, p. 69-86, 2005. 
SANTOS, José Manuel. Introdução à ética. Lisboa: Documenta, 2012.

SMUDDE, Peter M.; COURTRIGHT, Jeffrey L. A holistic approach to stakeholder management: a rhetorical foundation. Public Relations Review, Amsterdam, v. 37, n. 2, p. 137-144, jun. 2011.

STOKES, Ashli Q.; WAYMER, Damion. The good organization communicating well: teaching rhetoric in the public relations classroom. Public Relations Review, Amsterdam, v. 37, n. 5, p. 441-449, 2011.

SWEETSER, Kaye D. A losing strategy: the impact of nondisclosure in social media on relationships. Journal of Public Relations Research, Abingdon, v. 22, n. 3, p. 288-312, 2010.

VÁZQUEZ, Adolfo Sánchez. Ética. Rio de Janeiro: Civilização Brasileira, 2007.

WATSON, Tom. IPRA Code of Athens - The first international code of public relations ethics: its development and implementation since 1965. Public Relations Review, Amsterdam, v. 40, n. 4, p. 707-714, nov. 2014.

WHITE, Candace. Activist efforts of the Center for Media and Democracy to affect FCC policy for video news releases. Public Relations Review, Amsterdam, v. 38, n. 1, p. 76-82, mar. 2012.

WISE, Kurt. Lobbying and relationship management: the K Street connection. Journal of Public Relations Research, Abingdon, v. 19, n. 4, p. 357-376, 2007.

Texto recebido em 27.07.2017 e aprovado em 09.11.2017. 